\title{
Frontières
}

\section{Bibliographie indicative sur le deuil}

\section{Anne Létourneau}

Volume 16, numéro 2, printemps 2004

URI : https://id.erudit.org/iderudit/1074138ar

DOI : https://doi.org/10.7202/1074138ar

Aller au sommaire du numéro

Éditeur(s)

Université du Québec à Montréal

ISSN

1180-3479 (imprimé)

1916-0976 (numérique)

Découvrir la revue

Citer ce document

Létourneau, A. (2004). Bibliographie indicative sur le deuil. Frontières, 16(2), 107-107. https://doi.org/10.7202/1074138ar d'utilisation que vous pouvez consulter en ligne.

https://apropos.erudit.org/fr/usagers/politique-dutilisation/ 


\section{BIBLIOGRAPHIE INDICATIVE SUR LE DEUIL}

Anne Létourneau

ALEXANDER, H. (2001). Surmonter l'épreuve du deuil, Paris, Empreinte Temps Présent.

ALONZO, A.-M. (2001). Jamais de la vie: écrits et images sur les pertes et les deuils, Montréal, Éditions du Passage.

AURIACOMBE, E. (2001). Les deuils infantiles: effroi, indifférence, hantise, Paris, L'Harmattan.

BACQUÉ, M.-F. et M. HANUS (2001). Le deuil, Paris, Presses universitaires de France.

BACQUÉ, M.-F. (2003). Apprivoiser la mort: psychologie du deuil et de la perte, Paris, Odile Jacob.

BAUDRY, P. et H.-P. JEUDY (2001). Le deuil impossible, Paris, Eschel.

BEAUTHÉAC, N. (2002). Le deuil. Comment y faire face ? Comment le surmonter?, Paris, Seuil.

BERNARD, S. (2002). Et si la mort m'aidait à vivre?, Loretteville, Dauphin Blanc.

BROCA, A. (de) (2002). Douleurs, soins palliatifs, deuil, Paris, Masson.

CARPENTIER, M. (2002). Quelques mots sur une absence, Paris, L'Harmattan.

CENTRE DE PRÉVENTION DU SUICIDE ET D'INTERVENTION DE CRISE DU BAS-SAINT-LAURENT (2002). Phare dans la tempête: groupe de soutien pour les personnes endeuillées par suicide, Rimouski, Centre de prévention du suicide et d'intervention de crise du Bas-Saint-Laurent.

CORNILLOT, P. et M. HANUS (dir.) (2000). Parlons de la mort et du deuil, Paris, Frison-Roche.

COX, G.R. et al. (2001). Complicated grieving and bereavement: understanding and treating people experiencing loss, Amityville, Baywood.

DOKA, K. (dir.) (2002). Disenfranchised grief: new directions, challenges, and strategies for practice, Champaign, Research Press.

ERNOULT-DELCOURT, A. et D. DAVOUS (2001). Animer un groupe d'entraide pour personnes en deuil, Paris (Montréal), L'Harmattan.

FAURÉ, C. (1998). Vivre le deuil au jour le jour : réapprendre à vivre après la mort d'un proche, Paris, J'ai lu.

GAGNON, C. et DIOCÈSE DE SAINTE-ANNE-DE-LAPOCATIÈRE, SERVICE D'INITIATION CHRÉTIENNE (2002). La mort de Sébasse: pour accompagner son enfant lors de la mort d'un proche, La Pocatière, Service d'initiation chrétienne du diocèse de Sainte-Anne-de-la-Pocatière.

GAUGHEN, S. (dir.) (2003). Coping with death, San Diego, Greenhaven Press.

HANUS, M. (2001). La résilience, à quel prix? Survivre \& rebondir, Paris, Maloine.

HUNTLEY, T. (2002). Helping children grieve: when someone they love dies, Minneapolis, Augsburg Fortress.

ILSE, S. et A. APPLEBAUM (dir.) (2002). Empty arms: coping with miscarriage, stillbirth and infant death, Maple Plain, Wintergreen Press.

IRELAND, M. (2001). Apprivoiser le deuil : surmonter la perte d'un être cher, Paris, Presses du Châtelet.
JACQUES, J. (2002). Les saisons du deuil: la mort tisserande de la vie, Outremont, Quebecor.

JACQUET-SMAILOVIC, M. (2002). L'enfant, la maladie et la mort: la maladie et la mort d'un proche expliquées à l'enfant, Bruxelles, De Boeck. KEIRSE, M. (2000). Faire son deuil, vivre un chagrin: un guide pour les proches et les professionnels, Paris, De Boeck/Belin.

LEVY, A. (2000). Surmonter le deuil de ses parents, Paris, InterÉditions.

MARKHAM, U. (2001). Le deuil, Paris, J'ai lu.

MOREAU, E. (2001). Vivre le deuil, Paris, JacobDuvernet.

MORGAN, J. et P. LAUNGANI (dir.) (2002). Death and bereavement around the world, Amityville, Baywood Publishing.

MORGENTHALER, S. et D. MORGENTHALER (2001). Au jardin de ma mère: chronique d'un deuil, Strasbourg, La nuée bleue.

OPPENHEIM, D. (2002). Parents en deuil : Le temps reprend son cours, Ramonville-Saint-Agne, Érès.

OUAKNIN, J. (2002). L'âme immortelle: précis des lois et coutumes du deuil dans le judaïsme, Paris, Bibliophane, Consistoire de Paris.

POLETTI, R. et B. DOBBS (2001). Vivre le deuil en famille: des pistes pour traverser l'épreuve, SaintMaurice, Saint-Augustin.

POLETTI, R. et B. DOBBS (2003, nouvelle édition). Vivre son deuil et croître, Genève, Jouvence.

ROBITAILLE, M. (2003). La peine des sans-voix: l'accompagnement des déficients intellectuels en deuil, Lac-Beauport, Éditions Académie Impact.

SEIDEN, D.R. (2002). Always in my heart: a survivor's guide for coping with the loss of a spouse, Danbury, Rutledge Books.

STROEBE, M.S. (2001). Handbook of bereavement research: consequences, coping, and care, Washington, American Psychological Association.

THOMPSON, N. (dir.) (2002). Loss and grief: a guide for human service practitioners, Houndsmills, Palgrave.

WORDEN, J.W. (2002). Grief counseling and grief therapy: a help book for the mental health practitioner, New York, Springer.

ZONNEBELT-SMEENGE, S. J. et R. C. DE VRIES (2001). Surmontez la perte du conjoint: comment passer le cap de la douleur, Valence, Ligue pour la lecture de la bible.

ZUIDGEEST, P. (2001). The absence of God: exploring the Christian tradition in a situation of mourning, Leiden, E.J. Brill. 